\title{
Plane Sections of Convex Bodies of Maximal Volume*
}

\author{
E. Makai, ${ }^{1}$ S. Vrećica, ${ }^{2}$ and R. Živaljević ${ }^{3}$ \\ ${ }^{1}$ Mathematical Institute of the Hungarian Academy of Sciences, \\ H-1364 Budapest, PF. 127, Hungary \\ makai@math-inst.hu \\ ${ }^{2}$ Faculty of Mathematics, University of Belgrade, \\ Studentski trg 16, 11001 Belgrade, p.p. 550, Yugoslavia \\ vrecica@matf.bg.ac.yu \\ ${ }^{3}$ Mathematical Institute of the Serbian Academy of Sciences and Arts, \\ Knez Mihajlova 35/1, 11001 Belgrade, p.p. 367, Yugoslavia \\ rade@turing.mi.sanu.ac.yu
}

\begin{abstract}
Let $\mathcal{K}=\left\{K_{0}, \ldots, K_{k}\right\}$ be a family of convex bodies in $R^{n}, 1 \leq k \leq n-1$. We prove, generalizing results from [9], [10], [13], and [14], that there always exists an affine $k$-dimensional plane $A_{k} \subseteq R^{n}$, called a common maximal $k$-transversal of $\mathcal{K}$, such that, for each $i \in\{0, \ldots, k\}$ and each $x \in R^{n}$,

$$
V_{k}\left(K_{i} \cap A_{k}\right) \geq V_{k}\left(K_{i} \cap\left(A_{k}+x\right)\right),
$$
\end{abstract}

where $V_{k}$ is the $k$-dimensional Lebesgue measure in $A_{k}$ and $A_{k}+x$. Given a family $\mathcal{K}=\left\{K_{i}\right\}_{i=0}^{l}$ of convex bodies in $R^{n}, l<k$, the set $C_{k}(\mathcal{K})$ of all common maximal $k$-transversals of $\mathcal{K}$ is not only nonempty but has to be "large" both from the measure theoretic and the topological point of view. It is shown that $C_{k}(\mathcal{K})$ cannot be included in a $v$-dimensional $C^{1}$ submanifold (or more generally in an $\left(\mathcal{H}^{v}, v\right)$-rectifiable, $\mathcal{H}^{v}$-measurable subset) of the affine Grassmannian $A G r_{n, k}$ of all affine $k$-dimensional planes of $R^{n}$, of $O(n+1)$-invariant $v$-dimensional (Hausdorff) measure less than some positive constant $c_{n, k, l}$, where $v=(k-l)(n-k)$. As usual, the "affine" Grassmannian $A G r_{n, k}$ is viewed as a subspace of the Grassmannian $G r_{n+1, k+1}$ of all linear $(k+1)$-dimensional subspaces of $R^{n+1}$. On the topological side we show that there exists a nonzero cohomology class $\theta \in H^{n-k}\left(G_{n+1, k+1} ; Z_{2}\right)$ such that the class $\theta^{l+1}$ is concentrated in an arbitrarily small neighborhood of $C_{k}(\mathcal{K})$. As an immediate consequence we deduce that the Lyusternik-

\footnotetext{
* The research of the first author was (partially) supported by the Hungarian National Foundation for Scientific Research, Grant No. T-016094, and the research of the second and third authors was (partially) supported by the Serbian Science Foundation, Grants No. 04M01 and 04M03.
} 
Shnirel'man category of the space $C_{k}(\mathcal{K})$ relative to $G r_{n+1, k+1}$ is $\geq k-l$. Finally, we show that there exists a link between these two results by showing that a cohomologically "big" subspace of $G r_{n+1, k+1}$ has to be large also in a measure theoretic sense.

\section{Introduction}

A convex body $K \subseteq R^{n}$ is a compact convex set with nonempty interior. A maximal $k$-section of a convex body $K \subseteq R^{n}$ is a set $K \cap A_{k}$, where $A_{k} \subseteq R^{n}$ is an affine $k$-dimensional plane such that, for each $x \in R^{n}$,

$$
V_{k}\left(K \cap A_{k}\right) \geq V_{k}\left(K \cap\left(A_{k}+x\right)\right) .
$$

Here $V_{k}$ denotes the normalized Lebesgue measure defined on affine $k$-dimensional subspaces in $R^{n}$ (i.e., the measure of a unit cube is 1 ). Given a family $\mathcal{K}$ of convex bodies, a common maximal $k$-transversal of $\mathcal{K}$ is an affine $k$-dimensional plane $A_{k}$ for which $K \cap A_{k}$ is a maximal $k$-section of $K$ for each element $K$ of $\mathcal{K}$.

In this paper we give lower bounds on the size of the set $C_{k}(\mathcal{K})$ of all common maximal $k$-transversals of $\mathcal{K}$, both from the measure theoretic and topological point of view. It is obvious that $C_{k}(\mathcal{K})$ is "in general" empty if $|\mathcal{K}|>k+1$, where $|\mathcal{K}|$ is the cardinality of the family $\mathcal{K}$. We show that the condition $|\mathcal{K}| \leq k+1$ that is "in general" necessary for the existence of a common maximal $k$-transversal of $\mathcal{K}$ is also sufficient (Theorem 6). When the size of the family $\mathcal{K}$ decreases below $k+1$, we show that the set $C_{k}(\mathcal{K})$ increases both measure theoretically and topologically (Theorems 7 and 8). Theorem 9 is a link between these two results, which shows that a sufficiently cohomologically nontrivial compact $C^{1}$ submanifold (or more generally a compact $\left(\mathcal{H}^{v}, v\right)$-rectifiable, $\mathcal{H}^{\nu}$-measurable subset) of a Grassmannian must also have a sufficiently large measure in its own dimension. Our methods are: reduction to a topological coincidence question for a family of continuous cross sections of a vector-bundle, integral geometry in homogeneous spaces, topology of Grassmannians, cohomological technique, and Poincaré duality. Before we formulate precise statements of these theorems, we review some other related combinatorial geometric results which serve as a motivation for studying these questions.

\section{A Review of Motivating Results}

We always assume that $n \geq 2$. For $C \subseteq R^{n}$, we denote by $\operatorname{lin} C$ and aff $C$ the linear and affine subspace of $R^{n}$ spanned by $C$, respectively. $O(n)$ denotes the orthogonal group in $R^{n}$, that is, the group of all linear isometries of $R^{n}$, while $S O(n)$ is the special orthogonal group $\{T \in O(n) \mid \operatorname{det} T=1\}$. The line segment with endpoints $x$ and $y$ is denoted by $[x, y]$.

Now we recall some definitions, see also 2.10.1-2 of [6]. For a metric space $X$ and $m \geq 0$ the $m$-dimensional Hausdorff-measure $\mathcal{H}^{m}$ is an outer measure defined on all 
subsets of $X$ as follows: for $A \subset X$,

$$
\begin{gathered}
\mathcal{H}^{m}(A)=\sup _{\delta>0}\left(\operatorname { i n f } \left\{\sum_{i=1}^{\infty} \operatorname{diam}\left(A_{i}\right)^{m} \cdot \pi^{m / 2} /\left(2^{m} \Gamma\left(1+\frac{m}{2}\right)\right) \mid\right.\right. \\
\left.\left.A \subset \bigcup_{i=1}^{\infty} A_{i} \subset X, \forall i, \operatorname{diam}\left(A_{i}\right) \leq \delta\right\}\right),
\end{gathered}
$$

where diam means diameter. All closed subsets of $X$ are $\mathcal{H}^{m}$-measurable (see pp. 54 and 170 of [6]). If $m$ is a positive integer, one calls $A \subset X$, with $\mathcal{H}^{m}(A)<\infty,\left(\mathcal{H}^{m}, m\right)$ rectifiable, if,

$$
\forall \varepsilon>0, \quad \exists A_{\varepsilon} \subset X, \quad \mathcal{H}^{m}\left(A \backslash A_{\varepsilon}\right)<\varepsilon,
$$

and $A_{\varepsilon}$ is the image of a bounded subset of $R^{m}$ by a Lipschitz map defined on this subset, see pp. 251-252 of [6]. The zero-dimensional Hausdorff-measure $\mathcal{H}^{0}(A)$ of a set $A$ equals its cardinality, if $A$ is finite, and equals $\infty$ if $A$ is infinite. One calls $A \subset X\left(\mathcal{H}^{0}, 0\right)$-rectifiable if $A$ is finite [6, p. 252].

If $X$ is a Euclidean space and $A$ is a compact $C^{1} m$-dimensional submanifold, then $A$ is $\left(\mathcal{H}^{m}, m\right)$-rectifiable, and $\mathcal{H}^{m}(A)$ coincides with the differential geometric $m$-dimensional volume (Theorems 3.2.26 and 3.2.39 in [6]).

There are several star-shaped sets associated with a convex body $K \subseteq R^{n}$. For $0 \in$ int $K$ the double chordal symmetral $2 \tilde{\Delta} K$ of $K$ (see Definition 5.1.3 of [7]) is defined by $2 \tilde{\Delta} K:=\left\{\lambda u\left|u \in S^{n-1},\right| \lambda \mid \leq V_{1}(K \cap(R u))\right\}$, where $R u=\{r u \mid r \in R\} ; \tilde{\Delta} K$ is a 0 -symmetric star-shaped set having the same chord lengths through 0 in all directions as $K$. The difference body of $K$ (see $\S 12$, Section 53 of [1], or Section 3.2 of [7]) is $K+(-K)$; equivalently, it can be defined as $\left\{\lambda u\left|u \in S^{n-1},\right| \lambda \mid \leq \max \left\{V_{1}(K \cap(R u+x)) \mid x \in\right.\right.$ $\left.R^{n}\right\}$. The inclusion $2 \tilde{\Delta} K \subseteq K+(-K)$ readily follows from the definitions.

Theorem 1 [10, Theorem 3.1], [17, proof of Theorem 4]. For any convex body $K \subseteq$ $R^{n}$ with $0 \in$ int $K$ the boundaries of the associated double chordal symmetral and the difference body have a nonempty intersection:

$$
\text { bd }(2 \tilde{\Delta} K) \cap \operatorname{bd}(K+(-K)) \neq \varnothing
$$

Geometrically, this theorem says that 0 belongs to some affine diameter $[x, y]$ of $K$, that is, to a nondegenerate line segment $[x, y]=\operatorname{aff}\{x, y\} \cap K$ such that, for each $z \in R^{n}, \quad V_{1}([x, y]) \geq V_{1}(K \cap(\operatorname{aff}\{x, y\}+z))$. Equivalently, in a more usual formulation, there exist two different parallel supporting hyperplanes of $K$, passing through $x$ and $y$, respectively, see p. 293 of [10]. The geometrical reformulation of the statement of the theorem remains true even without the assumption $0 \in$ int $K$. A purely geometrical proof can be found in [10], while the proof in [17] uses elementary index theory as presented in [18]. Theorem 1 of [9] proves the geometrical reformulation in the case $0 \notin K$, but its arguments are easily modifiable to obtain the case $0 \in K$ as well.

For $0 \in$ int $K$ the intersection body $I K$ of $K$, introduced in [12] (see also Definition 8.1.1 of [7]), is defined by $I K:=\left\{\lambda u\left|u \in S^{n-1},\right| \lambda \mid \leq V_{n-1}\left(K \cap u^{\perp}\right)\right\}$, where $u^{\perp}=\left\{x \in R^{n} \mid\langle x, u\rangle=0\right\}$. The cross-section body $C K$ of $K$, introduced in [15] (see also Definition 8.3.1 of [7]) is defined by $C K:=\left\{\lambda u\left|u \in S^{n-1},\right| \lambda \mid \leq\right.$ 
$\left.\max \left\{V_{n-1}\left(K \cap\left(u^{\perp}+x\right)\right) \mid x \in R^{n}\right\}\right\}$. Again there is an inclusion of the form $I K \subseteq C K$ and a theorem relating the boundaries of these bodies.

Theorem 2 [13, Theorem 1 obtained jointly with R. Gardner]. For any convex body $K \subseteq R^{n}$ with $0 \in$ int $K$ one has bd $(I K) \cap$ bd $(C K) \neq \emptyset$.

Geometrically, this theorem says that 0 belongs to a maximal $(n-1)$-dimensional section $K \cap u^{\perp}$ of $K$, for some $u \in S^{n-1}$. In other words the section $K \cap u^{\perp}$ has the property that, for each $x \in R^{n}, V_{n-1}\left(K \cap u^{\perp}\right) \geq V_{n-1}\left(K \cap\left(u^{\perp}+x\right)\right)$. This geometrical reformulation holds even without the assumption $0 \in$ int $K$. Actually, not only must there exist some $u \in S^{n-1}$ for which $K \cap u^{\perp}$ is maximal in the sense above, but the set of all such unit vectors cannot be included in a compact $C^{1}(n-2)$-dimensional submanifold (or more generally in an $\left(\mathcal{H}^{n-2}, n-2\right)$-rectifiable, $\mathcal{H}^{n-2}$-measurable subset) of $S^{n-1}$, of $(n-2)$-volume (or more generally $(n-2)$-dimensional Hausdorff-measure) less than $c_{n-2}$, where $c_{n-2}$ is the volume of the sphere $S^{n-2}$. Moreover, here $c_{n-2}$ cannot be replaced by any larger number. It is implicit in the proof of Theorem 2 that there always exists a maximal $(n-1)$-section $K \cap u^{\perp}$ of $K$ such that $u^{\perp}$ contains any given linear $(n-2)$-dimensional subspace of $R^{n}$ prescribed in advance. The proof of this result is purely geometrical in combination with a simple continuity argument.

It was natural to try to generalize Theorems 1 and 2 both to the case of families of convex bodies and to the case of arbitrary $k$-dimensional maximal sections. As before $G r_{n, k}$ is the Grassmann manifold of all linear $k$-dimensional subspaces of $R^{n}$.

Theorem 3 [14, Theorem 4]. For any convex bodies $K_{0}, K_{1} \subseteq R^{n}$ there exists a line $A_{1} \subseteq R^{n}$ such that $K_{0} \cap A_{1}$ is an affine diameter of $K_{0}$ and $K_{1} \cap A_{1}$ is an affine diameter of $K_{1}$.

Theorem 4 [14, Theorem 3]. Let $1 \leq k \leq n-1$ be an integer. Then for any convex body $K \subseteq R^{n}$ the origin 0 belongs to some maximal $k$-section $K \cap A_{k}$ of $K$. Actually, the set of all such $k$-dimensional linear subspaces $A_{k}$ cannot be included in a $v$-dimensional compact $C^{1}$ submanifold (or, more generally, in an $\left(\mathcal{H}^{v}, v\right)$-rectifiable, $\mathcal{H}^{v}$-measurable subset) of the Grassmannian $G r_{n, k}$, of $O(n)$-invariant v-measure (or, more generally, $v$-dimensional Hausdorff measure) less than some positive constant $c_{n, k}$, where $v=$ $(k-1)(n-k)$. This is sharp in the sense that there exists some convex body $K$ such that the above set is a smooth compact $v$-dimensional submanifold of finite $O(n)$-invariant v-measure.

The $O(n)$-invariant $v$-dimensional Hausdorff measure $(v=(k-1)(n-k))$ is meant with respect to an $O(n)$-invariant Riemannian metric $d s^{2}$ on $G r_{n, k}$. This $O(n)$-invariant metric is described at the linear $k$-dimensional subspace $L_{k}^{0}=\operatorname{lin}\left\{e_{1}, \ldots, e_{k}\right\}$ spanned by the first $k$ basic vectors $e_{1}, \ldots, e_{k}$ as follows. The tangent space to $G r_{n, k}$ at the point $L_{k}^{0}$ is the set of all linear functions $A: \operatorname{lin}\left\{e_{1}, \ldots, e_{k}\right\} \rightarrow \operatorname{lin}\left\{e_{k+1}, \ldots, e_{n}\right\}$, and the Riemannian metric at $L_{k}^{0}$ is given by

$$
d s^{2}=\sum_{i=1}^{k} \sum_{j=k+1}^{n}\left\langle A e_{i}, e_{j}\right\rangle^{2}=\operatorname{Tr}\left(A^{*} \cdot A\right),
$$


where Tr means trace. Since $O(n)$ acts transitively on $G r_{n, k}$, this extends uniquely to a Riemannian metric on $G r_{n, k}$, by the formula that $d s^{2}$ at the left-translate $g^{\prime}(A)$ of $A$, for $g \in O(n)$, equals $d s^{2}$ at $A$, as given above. (Here $g^{\prime}$ denotes the derivative of the action by $g$, between the respective tangent spaces at $L_{k}^{0}$ and $g L_{k}^{0}$.) Still we have to see that this extension exists, i.e., if $g_{1}^{\prime}\left(A_{1}\right)=g_{2}^{\prime}\left(A_{2}\right)$, then $\operatorname{Tr}\left(A_{1}^{*} \cdot A_{1}\right)=\operatorname{Tr}\left(A_{2}^{*} \cdot A_{2}\right)$. Letting $g=g_{2}^{-1} g_{1}$, we have $g L_{k}^{0}=L_{k}^{0}$ and $A_{2}=g^{\prime}\left(A_{1}\right)$. That is, $g$ is the direct sum of two orthogonal matrices $h_{1}$ acting on $L_{k}^{0}$ and $h_{2}$ acting on $\operatorname{lin}\left\{e_{k+1}, \ldots, e_{n}\right\}$ and graph $A_{2}=g \cdot \operatorname{graph} A_{1}$, or $A_{2}=h_{2} A_{1} h_{1}^{-1}$. (For the relation about the graphs observe that at $L_{k}^{0}$ locally $G r_{n, k}$ can be considered as the vector space of all linear maps $L_{k}^{0} \rightarrow \operatorname{lin}\left\{e_{k+1}, \ldots, e_{n}\right\}$, by identifying these linear functions with their graphs. Then the action by $g$ becomes a linear map, whose derivative $g^{\prime}$ is a linear transformation, that pointwise coincides with $g$, after this identification.) $\operatorname{Then} \operatorname{Tr}\left(A_{2}^{*} \cdot A_{2}\right)=$ $\operatorname{Tr}\left(\left(h_{1}^{-1}\right)^{*} A_{1}^{*} h_{2}^{*} h_{2} A_{1} h_{1}^{-1}\right)=\operatorname{Tr}\left(\left(h_{1}^{-1}\right)^{*} A_{1}^{*} A_{1} h_{1}^{-1}\right)=\operatorname{Tr}\left(h_{1} A_{1}^{*} A_{1} h_{1}^{-1}\right)=\operatorname{Tr}\left(A_{1}^{*} A_{1}\right)$, as required. It is implicit in the proof of Theorem 4 that there always exists a maximal $k$-section $K \cap A_{k}$ of $K$ such that $A_{k}$ contains any linear $(k-1)$-dimensional subspace $L_{k-1}$ prescribed in advance.

Theorem 5 [14, Theorem 5]. For any convex bodies $K_{0}, \ldots, K_{n-1} \subseteq R^{n}$ there exists an affine $(n-1)$-dimensional plane $A_{n-1} \subseteq R^{n}$ such that for any $i \in\{0, \ldots, n-$ $1\}, K_{i} \cap A_{n-1}$ is a maximal $(n-1)$-section of $K_{i}$.

The proofs of Theorems 3-5 used partly geometrical arguments, sometimes involved ones, together with elementary topological tools like Grünbaum's theorem on the nonexistence of continuous even unit tangent vector fields on $S^{n-1}$, Brouwer's fixed point theorem, and the Borsuk-Ulam theorem on the nonexistence of odd continuous mappings $S^{n-1} \rightarrow S^{n-2}$.

Observe that the equivalent (geometrical) form of Theorem 1 which refers to the maximal 1-sections (without assuming $0 \in \operatorname{int} K$ ) is a special case of Theorem 3, when one body is chosen to be the unit ball centered at 0. Similarly, the geometrical form of Theorem 2 (again without assuming $0 \in$ int $K$ ) is a particular case of Theorem 4 (up to the value of the constant $c_{n, k}$ ). Moreover, the assertion after Theorem 2 about the existence of a maximal $(n-1)$-section $K \cap u^{\perp}$ of $K$ such that $u^{\perp}$ contains any linear $(n-2)$-dimensional subspace $L_{n-2} \subseteq R^{n}$, prescribed in advance, is a special case of Theorem 5 as well. Indeed, it is sufficient to apply Theorem 5 to the family $\left\{K, B_{1}, \ldots, B_{n-1}\right\}$, where $B_{i}$ are unit balls which have the property that the affine hull of their centers is equal to $L_{n-2}$.

The main objective of this paper is to extend Theorems 3-5 to the general case of $k$-sections, where $1 \leq k \leq n-1$.

\section{Statements of New Results}

In this section we formulate several results about common maximal $k$-sections for families of convex bodies in $R^{n}$. The first result is an existence result. It is shown that common maximal $k$-transversals exist if the given family $\mathcal{K}=\left\{K_{i}\right\}_{i=0}^{l}$ of convex bodies consists of $l+1 \leq k+1$ elements. The proof of this theorem is topological 
and follows from the assertion that $\left(w_{n-k}\right)^{k} \in H^{k(n-k)}\left(G r_{n, n-k} ; Z_{2}\right)$ is nonzero, where $w_{n-k}$ is the top Stiefel-Whitney class of the canonical $(n-k)$-plane bundle over $G r_{n, n-k}$. The following two theorems (Theorems 7 and 8) are of more quantitative nature. In Theorem 7 we show that measure theoretically the set $C_{k}(\mathcal{K})$ of all common maximal $k$-transversals of $\mathcal{K}$ is "sufficiently large" with respect to a measure defined on the Grassmannian $G r_{n+1, k+1}$. The argument is based on a generalized Cauchy-CroftonPoincaré formula from integral geometry. In Theorem 8 we show that the set $C_{k}(\mathcal{K})$ is cohomologically nontrivial in the following sense. There exists a cohomology class $\theta \in H^{n-k}\left(G r_{n+1, k+1} ; Z_{2}\right)$ such that for each open neighborhood $U$ of $C_{k}(\mathcal{K})$, the class $\theta^{l+1}$ is contained in the image of the homomorphism $H^{(l+1)(n-k)}(G r, G r \backslash U) \rightarrow$ $H^{(l+1)(n-k)}(G r)$, where $G r:=G r_{n+1, k+1}$ and the cohomology is taken with $Z_{2}$-coefficients. Finally, in Theorem 9 we establish a link between Theorems 7 and 8 by showing that any compact subset cohomologically nontrivial in the sense of Theorem 8 must be measure theoretically "large" in the sense of Theorem 7. This means that Theorem 8 is formally more general than Theorem 7. Note however that the key nontopological ideas in the proof of Theorem 9 are already present in the proof of Theorem 7, so this theorem can also be seen as a companion of Theorem 7 .

Theorem 6. Let $1 \leq k \leq n-1$ be an integer. Then for each family $\mathcal{K}=\left\{K_{0}, K_{1}, \ldots\right.$, $\left.K_{k}\right\}$ of convex bodies in $R^{n}$ there exists an affine common maximal $k$-transversal. In other words there exists an affine $k$-dimensional plane $A_{k}$ such that, for each $x \in R^{n}$ and each $i \in\{0, \ldots, k\}, V_{k}\left(K_{i} \cap A_{k}\right) \geq V_{k}\left(K_{i} \cap\left(A_{k}+x\right)\right)$, where $V_{k}$ is the normalized Lebesgue measure defined on affine $k$-dimensional subspaces of $R^{n}$.

Simple examples show that there exist families $\mathcal{K}=\left\{K_{i}\right\}_{i=0}^{k}$ of convex bodies in $R^{n}$ such that the common maximal $k$-section, whose existence was asserted in the previous theorem, is unique (see the remarks following Theorem 9). If the size of the family $\mathcal{K}$ is $l+1$, where $l<k$, then the set $C_{k}(\mathcal{K})$ of all common maximal $k$-sections is not only nonempty but is quite big both from a measure theoretic and a topological point of view. The exact statements are given in the following two theorems.

We assume that $R^{n}$ is embedded in the real projective space $R P^{n}$ in the usual way. Thus an affine $k$-dimensional plane $A_{k}$ of $R^{n}$ becomes a projective $k$-dimensional plane of $R P^{n}$, that can be identified with a linear $(k+1)$-dimensional plane of $R^{n+1}$ (namely, with $\operatorname{lin}\left(L_{k}+e_{n+1}\right)$, where $L_{k} \subseteq R^{n}$ is embedded in $R^{n+1}$ by $\left.\left(x_{1}, \ldots, x_{n}\right) \mapsto\left(x_{1}, \ldots, x_{n}, 0\right)\right)$. Thus the set of all affine $k$-dimensional planes of $R^{n}$ will be considered as a (dense open) subset of the Grassmannian $G r_{n+1, k+1}$, and the $O(n+1)$-invariant $(k-l)(n-k)$-measure is defined as the $(k-l)(n-k)$-dimensional Hausdorff-measure with respect to the $O(n+1)$-invariant Riemannian metric $d s^{2}$ on $G r_{n+1, k+1}$, defined like after Theorem 4 (for $n, k$ rather than $n+1, k+1$ ).

Theorem 7. Let $1 \leq k \leq n-1$ and $0 \leq l \leq k$ be integers and let $v:=(k-l)(n-k)$. Suppose that $\mathcal{K}=\left\{K_{0}, K_{1}, \ldots, K_{l}\right\}$ is a family ofl +1 convex bodies in $R^{n}$ and let $C_{k}(\mathcal{K})$ be the space of all common maximal $k$-transversals of $\mathcal{K}$. Then $C_{k}(\mathcal{K})$ cannot be included in an $\left(\mathcal{H}^{v}, v\right)$-rectifiable, $\mathcal{H}^{v}$-measurable subset of $G r_{n+1, k+1}$, of $O(n+1)$-invariant $v$ dimensional Hausdorff-measure less than some positive constant $c_{n, k, l}$. This is sharp in 
the sense that there are choices of $\mathcal{K}$ such that $C_{k}(\mathcal{K})$ is a compact $C^{\infty} v$-dimensional manifold of finite $O(n+1)$-invariant v-measure.

Definition 1. Let $K$ be a compact subset of a compact manifold $M$. We say that a cohomology class $\theta \in H^{p}\left(M ; Z_{2}\right)$ is concentrated in an arbitrarily small neighborhood of $K$ if, for each open neighborhood $U$ of $K$, the class $\theta$ is contained in the image of the map $H^{p}\left(M, M \backslash U ; Z_{2}\right) \rightarrow H^{p}\left(M ; Z_{2}\right)$.

Definition 2. Suppose that $A$ is a subspace of the topological space $X$. The LyusternikShnirel'man category $\operatorname{Cat}_{X}(A)$ of $A$ relative to $X$ is the minimum cardinality of a family $\mathcal{F}$ of closed sets such that $\bigcup \mathcal{F}=A$ and each element $F \in \mathcal{F}$ is contractible to a point by a homotopy that moves $F$ inside $X$.

As before, we consider the affine Grassmannian $A G r_{n, k}$ of all affine $k$-dimensional planes in $R^{n}$ as a subspace of the manifold of all $k$-dimensional planes in the projective space $R P^{n}$ which can be considered also as the Grassmannian $G r=G r_{n+1, k+1}$ of all linear $(k+1)$-dimensional subspaces of $R^{n+1}$.

Theorem 8. We make the same assumptions about $n, k, l$, and $\mathcal{K}$ as in Theorem 7. Given $v \in R^{n}$, let $\left[M_{v}\right]$ be the $Z_{2}$-fundamental class of the submanifold $M_{v}:=\{L \in$ $\left.A G r_{n, k} \mid v \in L\right\}$. Let $\theta \in H^{n-k}\left(G r_{n+1, k+1} ; Z_{2}\right)$ be the cohomology class which is the Poincaré dual of $\left[M_{v}\right]$. Then $\theta^{k+1} \neq 0$ and the class $\theta^{l+1}$ is concentrated in an arbitrarily small neighborhood of $C_{k}(\mathcal{K})$ in $G r_{n+1, k+1}$, in the sense of Definition 1.

Corollary 1. The Lyusternik-Shnirel'man category of the space $C_{k}(\mathcal{K})$ of all common maximal $k$-transversals relative to $G r:=G r_{n+1, k+1}$ is at least $k-l+1$,

$$
\mathrm{Cat}_{G r}\left(C_{k}(\mathcal{K})\right) \geq k-l+1 \text {. }
$$

Theorem 9. Suppose that $C \subseteq G r_{n+1, k+1}$ is a compact subset such that the class $\theta^{l+1} \in$ $H^{(n-k)(l+1)}\left(G r_{n+1, k+1} ; Z_{2}\right)$, defined in Theorem 8 , is concentrated in an arbitrarily small neighborhood of the subset $C$. Then the conclusion of Theorem 7 holds for the subset $C$. In other words, for $v=(k-l)(n-k)$, the $O(n+1)$-invariant v-measure of any $\left(\mathcal{H}^{v}, v\right)$-rectifiable, $\mathcal{H}^{v}$-measurable subset of $G r_{n+1, k+1}$ containing $C$ is at least $c_{n, k, l}$, where $c_{n, k, l}$ is the same constant as in Theorem 7.

Before turning to the proofs we make some remarks. For $l=k$, Theorem 7 reduces to Theorem 6. Theorem 6 is sharp in the sense that in general for $k+2$ convex bodies $K_{0}, \ldots, K_{k+1} \subseteq R^{n}$ there does not exist an affine $k$-dimensional plane $A_{k}$ such that $K_{i} \cap A_{k}$ is a maximal $k$-section of $K_{i}$ : take $k+2$ unit balls with centers not lying in an affine $k$-dimensional plane. Similarly, for $k+1$ convex bodies $K_{0}, \ldots, K_{k} \subseteq R^{n}$ in general there do not exist two affine $k$-dimensional planes with the stated property: take $k+1$ unit balls with centers not lying in an affine $(k-1)$-dimensional plane. Moreover, Theorem 6 includes Theorems 3 and 5, while Theorem 7 includes Theorem 4, by choosing $l=1, K_{0}=K, K_{1}$ the unit ball with center 0 . 


\section{Proofs of the Theorems}

Proof of Theorem 6. We begin with the observation that it suffices to prove the theorem for strictly convex bodies. Recall that a convex body $K$ is strictly convex if bd $K$ does not contain a line segment. This observation is based on the fact that each convex body can be approximated (in the sense of the Hausdorff distance) by strictly convex smooth $\left(C^{1}\right)$ bodies [1, §6, Section 27]. Also, if $K^{m} \subseteq R^{n}, m \in N$, is a sequence of convex bodies converging in the Hausdorff metric to a convex body $K \subseteq R^{n}$ and $A_{k}^{m} \subseteq R^{n}$ is a sequence of affine $k$-dimensional planes converging to an affine $k$-dimensional plane $A_{k} \subseteq R^{n}$ (in the topology inherited from $G r_{n+1, k+1}$ ) then, if $K^{m} \cap A_{k}^{m}$ is a maximal $k$-section of $K^{m}$ for all $m$, then $K \cap A_{k}$ is a maximal $k$-section of $K$. The details of this argument can be found in [14]. We conclude from here that the general result follows from the result in the special case of strictly convex bodies, so from hereon we assume that all bodies $K_{i}$ are strictly convex.

Our general plan of the proof is to reduce the problem of the existence of common maximal $k$-transversals to a topological coincidence question for a family of continuous cross sections of a vector bundle over a Grassmannian manifold. A similar plan was applied in [14] and to other combinatorial geometric problems in [20] and [4]. Let $L_{k} \subseteq R^{n}$ be a linear $k$-dimensional subspace in $R^{n}$. Then any $k$-dimensional affine subspace of $R^{n}$, parallel to $L_{k}$, is of the form $L_{k}+x$, where we may choose $x$ from the linear subspace $L_{k}^{\perp}$ that is the orthogonal complement of $L_{k}$. Let $\pi: R^{n} \rightarrow L_{k}^{\perp}$ be the orthogonal projection onto $L_{k}^{\perp}$. For $x \in \pi\left(K_{i}\right)$ let $f_{i}(x)=V_{k}\left(K_{i} \cap\left(L_{k}+x\right)\right)^{1 / k}$. By the Brunn-Minkowski theorem [1, $§ 11$, Section 48] $f_{i}$ is a concave function on $\pi\left(K_{i}\right)$. By strict convexity of $K_{i}$, for $x \in \operatorname{relbd}\left(\pi\left(K_{i}\right)\right)$ the intersection $K_{i} \cap\left(L_{k}+x\right)$ consists of one point, hence $f_{i}(x)=0$ for such $x$. (The relative boundary (relbd) and relative interior (relint) are taken with respect to $L_{k}^{\perp}$.) Hence $f_{i}(x)$ is a continuous function on $\pi\left(K_{i}\right)$ which attains its maximum in $\operatorname{relint}\left(\pi\left(K_{i}\right)\right)$, and for such a point of maximum $x$ we have that $K_{i} \cap\left(L_{k}+x\right)$ is a $k$-dimensional convex body.

Moreover, this maximum point $x$ is unique. Indeed, if there were two maximum points $x_{1}, x_{2} \in \operatorname{relint}\left(\pi\left(K_{i}\right)\right)$, then by concavity of $f_{i}$ on $\pi\left(K_{i}\right)$ we would have $f\left(x_{1}\right)=$ $f\left(\frac{1}{2}\left(x_{1}+x_{2}\right)\right)=f\left(x_{2}\right)$. From here and the well-known fact that the Brunn-Minkowski inequality is strict unless the convex bodies are homothetic (see $\S 11$, Section 48 of [1]), we deduce that $K_{i} \cap\left(L_{k}+x_{1}\right)$ and $K_{i} \cap\left(L_{k}+x_{2}\right)$ are translates of each other. Since $V_{k}\left(K_{i} \cap\left(L_{k}+x_{1}\right)\right)=V_{k}\left(K_{i} \cap\left(L_{k}+\frac{1}{2}\left(x_{1}+x_{2}\right)\right)\right)$, the inclusion $\frac{1}{2}\left[\left(K_{i} \cap\left(L_{k}+x_{1}\right)\right)+\right.$ $\left.\left(K_{i} \cap\left(L_{k}+x_{2}\right)\right)\right] \subseteq K_{i} \cap\left(L_{k}+\frac{1}{2}\left(x_{1}+x_{2}\right)\right)$ turns out to be an equality, hence also $K_{i} \cap\left(L_{k}+\frac{1}{2}\left(x_{1}+x_{2}\right)\right)$ is a translate of $K_{i} \cap\left(L_{k}+x_{1}\right)$. Therefore bd $K_{i}$ contains a line segment, contradicting strict convexity of $K_{i}$.

So we have a function $L_{k}^{\perp} \mapsto x_{i}\left(L_{k}^{\perp}\right)$ where $x_{i}\left(L_{k}^{\perp}\right)$ is the unique point where $f_{i}$ attains its maximum. It is not difficult to show that this function is continuous. Indeed, if $L_{k}^{m} \rightarrow L_{k}$ is a convergent sequence in $G r_{n, k}$, then any limit point of $x_{i}\left(\left(L_{k}^{m}\right)^{\perp}\right)$ equals $x_{i}\left(L_{k}^{\perp}\right)$ and all $x_{i}\left(\left(L_{k}^{m}\right)^{\perp}\right)$ lie in a compact set. This is sufficient to imply the continuity of the function $L_{k}^{\perp} \mapsto x_{i}\left(L_{k}^{\perp}\right)$. The details of the above arguments can be found in [14].

The theorem clearly follows if we can find a plane $L_{k} \in G r_{n, k}$ and some $x \in L_{k}^{\perp}$ such that $K_{i} \cap\left(L_{k}+x\right)$ is a maximal $k$-section of $K_{i}$ for each $i \in\{0, \ldots, k\}$. In other words we have to prove that for some $L_{k} \in G r_{n, k}$ we have a coincidence $x_{0}\left(L_{k}^{\perp}\right)=\cdots=x_{k}\left(L_{k}^{\perp}\right)$. Note that each of the functions $x_{i}$ can be considered as a continuous cross section of the 
canonical $(n-k)$-plane bundle over the Grassmann manifold $G r_{n, n-k}$. Recall that the total space of this bundle is $\left\{\left(L_{k}^{\perp}, x\right) \mid L_{k}^{\perp} \in G r_{n, n-k}, x \in L_{k}^{\perp} \subseteq R^{n}\right\}$ with the topology inherited from the product space $G r_{n, n-k} \times R^{n}$, and its projection is $\left(L_{k}^{\perp}, x\right) \mapsto L_{k}^{\perp}$. We consider the $k$ continuous cross sections $x_{1}\left(L_{k}^{\perp}\right)-x_{0}\left(L_{k}^{\perp}\right), \ldots, x_{k}\left(L_{k}^{\perp}\right)-x_{0}\left(L_{k}^{\perp}\right)$ of this bundle. By [5] or Proposition 2 of [20], any $k$ continuous cross sections of this bundle have a common zero. In other words, there exists a plane $L_{k}^{\perp} \in G r_{n, n-k}$ such that $x_{0}\left(L_{k}^{\perp}\right)=\cdots=x_{k}\left(L_{k}^{\perp}\right)$, which completes the proof of the theorem.

In the proof of the following theorem the integral geometric considerations, leading to the proof of $(3)$ in the $\left(\mathcal{H}^{v}, v\right)$-rectifiable, $\mathcal{H}^{v}$-measurable case, were kindly communicated to us by R. Howard.

Proof of Theorem 7. 1. We want to prove that the set $C_{k}(\mathcal{K})$ cannot be included in an $\left(\mathcal{H}^{v}, v\right)$-rectifiable, $\mathcal{H}^{v}$-measurable subset of $O(n+1)$-invariant $v$-dimensional Hausdorff measure smaller than a positive constant $c_{n, k, l}$. For $l=k$ the set $C_{k}(\mathcal{K})$ is not empty, an $\left(\mathcal{H}^{0}, 0\right)$-rectifiable set is just a finite set, and a zero-dimensional Hausdorff-measure of a finite set is just the cardinality of the set. Hence we may choose $c_{n, k, k}=1$. Now let $0 \leq l<k$. We choose $k-l$ additional bodies $K_{l+1}, \ldots, K_{k}$. It is convenient to choose these bodies to be unit balls such that their centers are affinely independent. Obviously each affine $(k-l-1)$-dimensional plane can arise as the affine hull of these centers, for an appropriate choice of these balls. Then by Theorem 6 there exists an affine $k$-dimensional plane $A_{k} \subseteq R^{n}$ such that for each $i \in\{0, \ldots, l\}$ we have that $K_{i} \cap A_{k}$ is a maximal $k$-section of $K_{i}$, and $A_{k}$ contains an arbitrary affine $(k-l-1)$-dimensional plane $A_{k-l-1}$ given in advance. Moreover, we may also take projective $(k-l-1)$-dimensional planes $P_{k-l-1}$ rather than affine ones. In fact, if a projective $(k-l-1)$-dimensional plane $P_{k-l-1}$ lies in the infinite hyperplane of $R^{n}$, e.g., is the infinite hyperplane of $\operatorname{lin}\left\{e_{1}, \ldots, e_{k-l}\right\}$, then we choose the centers of the unit balls $K_{l+1}, \ldots, K_{k}$ at $\lambda e_{1}, \ldots, \lambda e_{k-l}$, and let $\lambda \rightarrow \infty$. Again by Theorem 6 there exist affine $k$-dimensional planes $A_{k}(\lambda)$ which give maximal $k$-sections of all bodies $K_{0}, \ldots, K_{l}$ and which converge to a (projective) $k$-dimensional plane $A_{k}$ that necessarily contains $P_{k-l-1}$. However, then for each $i \in\{0, \ldots, l\}$ we have that $K_{i} \cap A_{k}$ is a maximal $k$-section of $K_{i}$ (see [14], cited in the beginning of the proof of Theorem 6 ).

For a projective $(k-l-1)$-dimensional plane $P_{k-l-1} \subseteq R P^{n}$ (real projective $n$ dimensional space) let

$$
S_{k}\left(P_{k-l-1}\right)=\left\{P_{k} \mid P_{k} \subseteq R P^{n} \text { is a projective } k \text {-dimensional plane, } P_{k-l-1} \subseteq P_{k}\right\} .
$$

Recall that $C_{k}(\mathcal{K})=\left\{A_{k} \mid A_{k} \subseteq R^{n}\right.$ is an affine $k$-dimensional plane such that $(\forall i \in$ $\{0, \ldots, l\}) K_{i} \cap A_{k}$ is a maximal $k$-section of $\left.K_{i}\right\}$. Henceforward we consider $C_{k}(\mathcal{K})$ as a set of projective $k$-dimensional planes. Then the above assertion about projective $(k-l-1)$-dimensional planes can be reformulated as follows:

$$
\begin{aligned}
& \text { for each projective }(k-l-1) \text {-dimensional plane } P_{k-l-1} \subseteq R P^{n}, \\
& \qquad C_{k}(\mathcal{K}) \cap S_{k}\left(P_{k-l-1}\right) \neq \emptyset .
\end{aligned}
$$

The set $C_{k}(\mathcal{K})$ of affine, thus projective, $k$-dimensional planes in $R^{n}$ is considered as a subset of $G r_{n+1, k+1}$, and we identify the projective $(k-l-1)$-dimensional plane $P_{k-l-1}$ 
with a linear $(k-l)$-dimensional subspace $L_{k-l}$ of $R^{n+1}$. Moreover, we write $S_{k}\left(P_{k-l-1}\right)$ as $S_{k+1}\left(L_{k-l}\right)$, and we consider it as a set of linear $(k+1)$-dimensional subspaces of $R^{n+1}$. Thus

$$
\text { for each } L_{k-l} \in G r_{n+1, k-l}, \quad C_{k}(\mathcal{K}) \cap S_{k+1}\left(L_{k-l}\right) \neq \emptyset \text {. }
$$

Now we recall some integral geometric considerations from [3] and [11]. We follow the notation of [11] but assume that the results hold in the generality given in [3]. Let $G$ be a Lie group, let $K \subseteq G$ be a compact (thus Lie) subgroup of $G$, and let $G / K$ be the homogeneous space of left cosets $\xi K$ of $K$ in $G$. Then $G$ acts on $G / K$ by $g(\xi K)=(g \xi) K$. Let $\pi: G \rightarrow G / K$ denote the natural projection, whose derivative will be denoted by $\pi^{\prime}$. Suppose that $G$ has a Riemannian metric $d s^{2}$, that is left invariant on $G$, moreover is right invariant under elements of $K$. We denote the corresponding bilinear form on $G$ by $\langle$,$\rangle . This induces a G$-invariant Riemannian metric $d s^{\prime 2}$ on $G / K$. The corresponding bilinear form is denoted by $\langle,\rangle^{\prime}$, and it is described as follows:

$$
\langle X, X\rangle_{T(G / K)_{x}}^{\prime}=\left\langle\left[\pi^{\prime} \mid\left(\operatorname{ker} \pi^{\prime}(\xi)\right)^{\perp}\right]^{-1} X,\left[\pi^{\prime} \mid\left(\operatorname{ker} \pi^{\prime}(\xi)\right)^{\perp}\right]^{-1} X\right\rangle_{T G_{\xi}},
$$

where $x \in G / K$, and $\xi$ is any element of $\pi^{-1}(x)$. (Observe that $\pi^{\prime} \mid\left(\operatorname{ker} \pi^{\prime}(\xi)\right)^{\perp}$ is a linear isomorphism of the orthogonal complement $\left(\operatorname{ker} \pi^{\prime}(\xi)\right)^{\perp}$ of $\operatorname{ker} \pi^{\prime}(\xi)$ in $T G_{\xi}$ onto $T(G / K)_{x}$, and that this definition is independent of the choice of $\xi$.)

Let $\mathcal{H}^{p}$ be the $p$-dimensional Hausdorff measure on $G / K$ and let $M$ be an $\left(\mathcal{H}^{p}, p\right)$ rectifiable subset of $G / K$. (See Section 3.2.14 of [6] or Section 2 of this paper for the definition.) Basically these are subsets of finite $\mathcal{H}^{p}$ measure that can be covered up to a set of $\mathcal{H}^{p}$ zero by a countable number of Lipschitz images of subsets of $R^{p}$. This includes the class of rectifiable sets (which are the Lipschitz images of bounded subsets of $R^{p}$ ). If $M$ is also $\mathcal{H}^{p}$ measurable, then (see Theorem 3.2.19 of [6]) for $\mathcal{H}^{p}$ almost all $x \in M$ there is a $p$-dimensional subspace tangent space $\operatorname{Tan}^{p}\left(\mathcal{H}^{p}\lfloor M, x)\right.$ which we just denote by $T(M)_{x}$ and its orthogonal complement in $T(G / K)_{x}$ will be denoted by $T^{\perp}(M)_{x}$. (To simplify notation we sometimes suppress the dimension and just say that $M$ is $\mathcal{H}$ rectifiable and measurable.) If $M$ is an embedded $p$-dimensional submanifold of class $C^{1}$ and finite volume, then $M$ is $\mathcal{H}$ rectifiable and measurable and $\operatorname{Vol}(M)=\mathcal{H}^{p}(M)$.

Let $M \subset G / K$ be an $\left(\mathcal{H}^{p}, p\right)$-rectifiable subset of $G / K$ which is $\mathcal{H}^{p}$ measurable and let $N \subset G / K$ be an $\left(\mathcal{H}^{q}, q\right)$-rectifiable subset of $G / K$ which is $\mathcal{H}^{q}$-measurable and $p+q \geq \operatorname{dim}(G / K)$. Set $m=\operatorname{dim}(G / K)$. Then for almost all $g \in G$ the intersection $M \cap g N$ is an $\mathcal{H}^{p+q-m}$-measurable $\left(\mathcal{H}^{p+q-m}, p+q-m\right)$-rectifiable subset of $G / K$. Moreover, if $G$ is unimodular (in particular, compact, see Remark 2.4 of [11]), then the integral geometric formula, the so-called Poincaré's formula (compare Theorem 3.8 of [11] stated for the smooth case),

$$
\int_{G} \mathcal{H}^{p+q-n}(M \cap g N) d g=\iint_{M \times N} \sigma_{K}\left(T^{\perp}(M)_{x}, T^{\perp}(N)_{y}\right) d \mathcal{H}^{p} \times d \mathcal{H}^{q}(x, y)
$$

holds. Here $d g$ is the volume form on $G$ associated with the Riemannian metric $d s^{2}$ on $G$, see Remark 2.6 of [11], and the Hausdorff measures are taken with respect to the Riemannian metric $d s^{\prime 2}$. Further, $\sigma_{K}\left(T^{\perp}(M)_{x}, T^{\perp}(N)_{y}\right)$ (see Definition 3.3 of [11]) is a type of "integrated absolute value of sine of angle" and for our purposes all that 
matters is that $0 \leq \sigma_{K}\left(T^{\perp}(M)_{x}, T^{\perp}(N)_{y}\right) \leq \operatorname{Vol}(K)(\operatorname{Vol}(K)$ taken in the sense of the Riemannian metric $d s^{2}$, see the inequality $0 \leq \sigma(V, W) \leq 1$ from 2.1 of [11]) so that

$$
\int_{G} \mathcal{H}^{p+q-m}(M \cap g N) d g \leq \operatorname{Vol}(K) \cdot \mathcal{H}^{p}(M) \cdot \mathcal{H}^{q}(N) .
$$

This can be seen by modifying the arguments in either [3] or [11]. We briefly indicate how to do the modifications of the arguments of [11]. First note that the proof of the Basic Integral Formula ([11, p. 7]; assuming $G$ is unimodular this is essentially the special case of the formula above where $K=\{e\}$ is the trivial subgroup of $G$ ) only depends on Sard's theorem, the coarea formula, and some changes of variables in integrals. If in deriving formula (2-12) on p. 8 of [11] the smooth coarea formula is replaced by the form given by Federer (see Theorem 3.2.22, p. 258, of [6]) we get the same formula except that now $M$ and $N$ are as above and (using the notation of $[11, \mathrm{p} .8]$ ) the fibers $f^{-1}[g]$ are $H^{p+q-n}$-measurable, $\left(\mathcal{H}^{p+q-n}, p+q-n\right)$-rectifiable subsets of $G$ for almost all $g \in G$. The rest of the proof is pretty much word for word the same. Therefore the Basic Integral Formula of [11] holds when $M$ and $N$ are $\mathcal{H}$-rectifiable and measurable subsets of $G$. Poincaré's formula [11, Theorem 3.8, p. 15] follows from the Basic Integral Formula by purely formal considerations (basically changes of variables in integrals) so it will also hold for $\mathcal{H}$-rectifiable and measurable sets $M$ and $N$.

We apply this inequality to $G=S O(n+1), K=[S O(k+1) \times S O(n-k)] \cup$ $[(O(k+1) \backslash S O(k+1)) \times(O(n-k) \backslash S O(n-k))]$, where $O(k+1)$ acts on $\operatorname{lin}\left\{e_{1}, \ldots, e_{k+1}\right\}$ and $O(n-k)$ acts on $\operatorname{lin}\left\{e_{k+2}, \ldots, e_{n+1}\right\}$, where $\left\{e_{1}, \ldots, e_{n+1}\right\}$ is the standard orthonormal base of $R^{n+1}$. Then $K$ is the stabilizer of $\operatorname{lin}\left\{e_{1}, \ldots, e_{k+1}\right\}$ in $S O(n+1)$, hence $G / K$ can be identified with $G r_{n+1, k+1}$, with $\pi: G \rightarrow G / K$ given by $\pi(g)=$ $g\left(\operatorname{lin}\left\{e_{1}, \ldots, e_{k+1}\right\}\right)$ for $g \in S O(n+1)$.

We define the bi-invariant Riemannian metric $d s^{2}$ on $G$ as follows. We have that $T G_{I}$, the tangent space of $G$ at $I$, i.e., the Lie algebra of $G$, is the set of all skew-symmetric $n+1$ by $n+1$ matrices. Then for $T G_{I} \ni\left(a_{i j}\right)$ we let $d s^{2}=\sum_{i<j} a_{i j}^{2}=\frac{1}{2} \operatorname{Tr}\left[\left(a_{i j}\right)^{*}\left(a_{i j}\right)\right]$. This has a left-invariant and a right-invariant extension to $G$, which however coincide, to give a bi-invariant extension [11,3.12a].

Next we consider the derivative of $\pi$ at $I$, i.e., $\pi^{\prime}(I): T G_{I} \rightarrow T(G / K)_{\pi(I)}$. We recall from the remarks following Theorem 4 that $T(G / K)_{\pi(I)}$ is the set of all linear transformations $\operatorname{lin}\left\{e_{1}, \ldots, e_{k+1}\right\} \rightarrow \operatorname{lin}\left\{e_{k+2}, \ldots, e_{n+1}\right\}$. Then we have for $\left(a_{i j}\right) \in T G_{I}$ that $\pi^{\prime}(I)\left(a_{i j}\right)=\left(a_{i j}\right)_{\substack{1 \leq j \leq k+1 \\ k+2 \leq i \leq n+1}} \in T(G / K)_{\pi(I)}$. Therefore ker $\pi^{\prime}(I)=\left\{\left(a_{i j}\right) \in T G_{I} \mid\right.$ $a_{i j}=0$ for $\left.j \leq k+1<i\right\}$. Hence for its orthogonal complement $\left(\operatorname{ker} \pi^{\prime}(I)\right)^{\perp}$ in $T G_{I}$ in the sense of the above Riemannian metric $d s^{2}$ we have $\left(\operatorname{ker} \pi^{\prime}(I)\right)^{\perp}=\left\{\left(a_{i j}\right) \in T G_{I} \mid\right.$ $a_{i j}=0$ for $i, j \leq k+1$ and for $\left.i, j>k+1\right\}$.

By definition, $d s^{\prime 2}$ in $G / K$, at $\left(a_{i j}\right)_{k+2 \leq i \leq n+1}^{1 \leq j \leq k+1} \in T(G / K)_{\pi(I)}$ is obtained the following way. We extend $\left(a_{i j}\right)_{\substack{1 \leq j \leq k+1 \\ k+2 \leq i \leq n+1}}$ to an $n+1$ by $n+1$ matrix $\left(a_{i j}\right) \in\left(\operatorname{ker} \pi^{\prime}(I)\right)^{\perp}$, i.e., to a skew-symmetric matrix satisfying $a_{i j}=0$ for $i, j \leq k+1$ and for $i, j>k+1$, and evaluate $d s^{2}$ for the obtained matrix. This however gives us $\sum_{i<j} a_{i j}^{2}=\sum_{i>j} a_{i j}^{2}=$ $\sum_{i=k+2}^{n+1} \sum_{j=1}^{k+1} a_{i j}^{2}$. That is, on $T(G / K)_{\pi(I)}$ the $S O(n+1)$-invariant Riemannian metric $d s^{\prime 2}$ on $G / K=G r_{n+1, k+1}$ considered in [11] equals the $O(n+1)$-invariant Riemannian 
metric $d s^{2}$ on $G r_{n+1, k+1}$, introduced after Theorem 4 (for $n, k$ rather than $n+1, k+1$ ), and considered in this theorem. Since both of these Riemannian metrics are $S O(n+1)$ invariant, they coincide on the whole $G / K$.

Now suppose that $C_{k}(\mathcal{K})$ is included in an $\left(\mathcal{H}^{v}, v\right)$-rectifiable, $\mathcal{H}^{v}$-measurable subset $M$ of $G r_{n+1, k+1}$, where $v=(k-l)(n-k)$. We are going to show that the $O(n+1)$ invariant $v$-dimensional Hausdorff-measure of $M$, in the sense of the $O(n+1)$-invariant Riemannian metric $d s^{\prime 2}$ on $G r_{n+1, k+1}$ considered in this theorem, is at least a suitably chosen positive constant $c_{n, k, l}$.

By (2) we have for each $L_{k-l} \in G r_{n+1, k-l}$ that $M \cap S_{k+1}\left(L_{k-l}\right) \neq \emptyset$. Observe that for any $L_{k-l} \in G r_{n+1, k-l}$ we have that $S_{k+1}\left(L_{k-l}\right)$ is a compact $C^{\infty}$ manifold, diffeomorphic to $G r_{n-k+l+1, l+1}$, that has dimension $(l+1)(n-k)$. We fix some $L_{k-l}^{0} \in G r_{n+1, k-l}$. We have for any $g \in S O(n+1)$ that $S_{k+1}\left(g L_{k-l}^{0}\right)=g S_{k+1}\left(L_{k-l}^{0}\right)$. Let $N=S_{k+1}\left(L_{k-l}^{0}\right)$. Then for each $g \in S O(n+1)$ we have $M \cap g N=M \cap g S_{k+1}\left(L_{k-l}^{0}\right)=M \cap S_{k+1}\left(g L_{k-l}^{0}\right) \neq \emptyset$. Further, $v+\operatorname{dim} N=(k-l)(n-k)+(l+1)(n-k)=(k+1)(n-k)=\operatorname{dim} G r_{n+1, k+1}=$ $\operatorname{dim}(G / K)$.

Applying the above cited results from [11], for almost all $g \in S O(n+1)$ we have that $M \cap g N$ is a finite subset of $G r_{n+1, k+1}$, and we have by (3)

$$
\int_{S O(n+1)} \mathcal{H}^{0}(M \cap g N) d g \leq \operatorname{Vol}(K) \cdot \mathcal{H}^{\nu}(M) \cdot \mathcal{H}^{(l+1)(n-k)}(N),
$$

where

$K=[S O(k+1) \times S O(n-k)] \cup[(O(k+1) \backslash S O(k+1)) \times(O(n-k) \backslash S O(n-k))]$.

Since for almost all $g \in S O(n+1)$ we have that $M \cap g N$ is a finite set, in which case $1 \leq \mathcal{H}^{0}(M \cap g N)$, we have

$$
\operatorname{Vol}(S O(n+1)) \leq \int_{S O(n+1)} \mathcal{H}^{0}(M \cap g N) d g .
$$

The inequalities (4) and (5) together imply

$$
\mathcal{H}^{v}(M) \geq c_{n, k, l},
$$

for a suitably chosen positive constant $c_{n, k, l}$, as asserted.

2. It remains to be shown that, for some choice of the convex bodies $K_{0}, \ldots, K_{l}$ in $R^{n}$, the set $C_{k}(\mathcal{K})$ of affine $k$-dimensional planes $A_{k} \subseteq R^{n}$ in question is a compact $C^{\infty} v$ dimensional submanifold of $G r_{n+1, k+1}$, of the considered $O(n+1)$-invariant $\nu$-measure some finite number, for $v=(k-l)(n-k)$.

Let $K_{0}, \ldots, K_{l} \subseteq R^{n}$ be unit balls, whose centers span an affine $l$-dimensional plane $A_{l}^{0} \subseteq R^{n}$. Then $C_{k}(\mathcal{K})=S_{k}\left(A_{l}^{0}\right)=S_{k+1}\left(L_{l+1}^{0}\right)$, where $L_{l+1}^{0}$ is the linear $(l+1)$-dimensional subspace of $R^{n+1}$, corresponding to the affine (thus projective) $l$-dimensional plane $A_{l}^{0}$. Further $S_{k+1}\left(L_{l+1}^{0}\right)$ is diffeomorphic to $G r_{n-l, k-l}$, therefore $S_{k+1}\left(L_{l+1}^{0}\right)$ is a compact $C^{\infty} v$-dimensional submanifold of $G r_{n+1, k+1}$, hence has a finite $O(n+1)$-invariant $\nu$-measure.

Remark. Using [11] it is not difficult to give the constant $c_{n, k, l}$ from the above proof explicitly also for $l<k$. In fact, (4) and (5) give $\mathcal{H}^{\nu}(M) \geq \operatorname{Vol}(S O(n+1)) /[\operatorname{Vol}(K)$. 
$\operatorname{Vol}(N)]$. Here $\operatorname{Vol}(K)=2 \operatorname{Vol}(S O(k+1)) \cdot \operatorname{Vol}(S O(n-k))$. Moreover, we have $N=S_{k+1}\left(L_{k-l}^{0}\right) \cong G r_{n-k+l+1, l+1}$. Further, like in 3.12.(a) of [11], since $d s^{2}$ is biinvariant on $G$ and $\pi$ is a Riemannian submersion, we have $\operatorname{Vol}\left(G r_{n-k+l+1, l+1}\right)=$ $\operatorname{Vol}(S O(n-k+l+1)) /[2 \operatorname{Vol}(S O(l+1)) \cdot \operatorname{Vol}(S O(n-k))]$. It remains to observe that by 3.12(a), formula (3-15), of [11] for any natural number $m$ we have $\operatorname{Vol}(S O(m))=$ $\operatorname{Vol}\left(S^{1}\right) \operatorname{Vol}\left(S^{2}\right) \cdots \operatorname{Vol}\left(S^{m-1}\right)$.

Proof of Theorem 8. Like in Theorem 6, it also suffices to prove this theorem for strictly convex bodies only. Namely, let $K_{0}^{m}, \ldots, K_{l}^{m}, m \in N$, be strictly convex bodies converging (in the sense of the Hausdorff distance) to $K_{0}, \ldots, K_{l}$. As in the beginning of the proof of Theorem 6 , by the arguments in [14], for $\mathcal{K}^{m}=\left\{K_{0}^{m}, \ldots, K_{l}^{m}\right\}$ we have for any subsequence $m_{i} \in N$ that $A_{k}^{m_{i}} \in C_{k}\left(\mathcal{K}^{m_{i}}\right), A_{k}^{m_{i}} \rightarrow A_{k}$ imply $A_{k} \in C_{k}(\mathcal{K})$. Therefore $C_{k}\left(\mathcal{K}^{m}\right)$ (which are contained in a compact subset of $G r_{n+1, k+1}$ independent of $m$ ), lie, for $m$ sufficiently large, in an arbitrarily small open neighborhood of $C_{k}(\mathcal{K})$, prescribed in advance.

Next we recall some well-known facts about the topology of Grassmannians. Standard references are [16], [8], and [2] as well as other more elementary topological textbooks. We include the relevant details to make the proof as self-contained as possible.

The direct sum decomposition $R^{n+1} \cong R^{n} \oplus R$ and the identification of $R^{n}$ with the hyperplane $H:=\left\{x \in R^{n+1} \mid x_{n+1}=1\right\}$ allows us to view the affine Grassmann manifold $A G r_{n, k}$ of all affine $k$-dimensional planes in $R^{n}$ as a submanifold of $G r_{n+1, k+1}$. More precisely, the manifold $A G r_{n, k}$ is embedded in $G r_{n+1, k+1}$ by the map which sends a $k$-dimensional plane $L \in A G r_{n, k}$ to the $(k+1)$-dimensional linear subspace of $R^{n+1}$ spanned by $L$. From now on, $A G r_{n, k}$ will often be identified with its image in $G r_{n+1, k+1}$. The space $N_{0}:=G r_{n+1, k+1} \backslash A G r_{n, k}$ is identified as the Grassmann manifold $G r_{n, k+1}$ of all "horizontal" $(k+1)$-dimensional planes in $G r_{n+1, k+1}$. The "dual" manifold $N_{1}$ of all "vertical" $(k+1)$-dimensional planes in $G r_{n+1, k+1}$, i.e., the planes which contain the basic vector $e_{n+1}$, is clearly homeomorphic to $G r_{n, k}$. Indeed, each vertical $(k+1)$-dimensional plane $L$ in $R^{n+1}=R^{n} \oplus R$ intersects the horizontal space $R^{n}$ in a $k$-dimensional plane $L^{\prime}$ and conversely each $L^{\prime} \in G r_{n, k}$ determines the corresponding $L \in N_{1}$. A very important observation, already used in the proof of Theorem 6 , is that the space

$$
A G r_{n, k}=G r_{n+1, k+1} \backslash G r_{n, k+1}
$$

of all nonhorizontal $(k+1)$-dimensional linear subspaces of $R^{n+1}$ is homeomorphic to the total space of the canonical (tautological) $(n-k)$-dimensional bundle $\xi_{n-k}$ over $G r_{n, k} \cong$ $G r_{n, n-k}$. Recall that a $k$-dimensional plane $P \in A G r_{n, k}$ is of the form $P=L_{k}+e_{n+1}+v$ where $L_{k}$ is the unique parallel horizontal $k$-dimensional linear subspace in $R^{n}$ and $v \in$ $R^{n}$. Note that there exists a unique vector $v \in L_{k}^{\perp}$ in the linear subspace of $R^{n}$ orthogonal to $L_{k}$ such that $P=L_{k}+e_{n+1}+v$. This defines a bijective correspondence $P \mapsto\left(L_{k}, v\right)$ which proves the assertion that $A G r_{n, k}$ is a tautological, $(n-k)$-dimensional bundle over $G r_{n, k}$. Note that the zero section of this bundle is naturally identified with the manifold $N_{1}$. This follows from the fact that the zero section consists of the planes $P=\left(L_{k}, v\right)$ for which $v=0$. Note that this implies that $A G r_{n, k}=G r_{n+1, k+1} \backslash G r_{n, k+1}$ is an open "tubular" neighborhood of $N_{1}$ which can also be seen as the normal bundle $v\left(N_{1}\right)$ to $N_{1}$ in $G r_{n+1, k+1}$. All the (co)homologies are understood to be with $Z_{2}$ coefficients. Let 
[ $\left.N_{1}\right]$ be the fundamental class of $N_{1}$, i.e., the generator of the group $H_{k(n-k)}\left(N_{1}\right) \cong$ $H_{k(n-k)}\left(G r_{n, k}\right) \cong Z_{2}$. By a slight abuse of notation, let $\left[N_{1}\right]$ also be the image of this class in $H_{k(n-k)}\left(G r_{n+1, k+1}\right)$ upon the embedding $N_{1} \rightarrow G r_{n+1, k+1}$. The Poincaré dual of the class $\left[N_{1}\right]$, denoted by $\theta$, is a cohomology class in $H^{n-k}\left(G r_{n+1, k+1}\right)$. Actually, by the usual link between the Poincaré duality and the Thom isomorphism theorem, see, e.g., [2] or Theorem 10.2, Chapter VI, especially Section 11, of [16], $\theta$ can be seen as a class in $H^{*}\left(v\left(N_{1}\right), v\left(N_{1}\right) \backslash N_{1}\right) \cong H^{*}\left(G r_{n+1, k+1}, G r_{n+1, k+1} \backslash G r_{n, k+1}\right)$. Moreover, the Thom isomorphism theorem says that each class $\omega \in H^{*}\left(v\left(N_{1}\right), v\left(N_{1}\right) \backslash N_{1}\right)$ has a unique decomposition of the form $\omega=w \theta$ where $w \in H^{*}\left(v\left(N_{1}\right)\right) \cong H^{*}\left(N_{1}\right)$. In particular $\theta^{2}=w_{n-k} \theta$ where $w_{n-k}$ is the top Stiefel-Whitney class of the bundle $v\left(N_{1}\right) \rightarrow N_{1}$ or equivalently the $Z_{2}$-Euler class of the bundle $\xi_{n-k}$ over $G r_{n, k}$ defined above. Hence,

$$
\theta^{k+1}=\left(w_{n-k}\right)^{k} \theta .
$$

It is a well-known fact, already used in the proof of Theorem 6 , that $\left(w_{n-k}\right)^{k} \neq 0$. A cohomological proof of this observation can be found in [5], see also [20], while an alternative proof, based on Schubert calculus, is in Section 1.5 of [8]. From here we deduce that $\theta^{k+1}=w_{n-k}^{k} \theta \neq 0$ which follows from $w_{n-k}^{k} \neq 0$.

Let $x_{i}: G r_{n, n-k} \rightarrow \xi_{n-k}, i=0, \ldots, l$, be the sections of the bundle $\xi_{n-k} \cong v\left(N_{1}\right) \cong$ $A G r_{n, k}$, introduced in the proof of Theorem 6. Recall that $x_{i}\left(L_{k}^{\perp}\right)=x_{i}^{\prime}\left(L_{k}\right)$ is by definition the unique point in $L_{k}^{\perp}$ such that $L_{k}+x_{i}\left(L_{k}^{\perp}\right)$ is the maximal $k$-section of the strictly convex body $K_{i}$. Let $X_{i}=\operatorname{Im}\left(x_{i}\right)$, considered as a subset of $\xi_{n-k} \subset G r_{n+1, k+1}$. By definition, $C_{k}(\mathcal{K})=\bigcap_{i=0}^{l} X_{i}$. Note that $X_{i}$ is a $C^{0}$ submanifold of $\xi_{n-k}$ since $x_{i}$ is a continuous cross section, and that the fundamental class $\left[X_{i}\right]$ of $X_{i}$, seen as a class in $H^{*}\left(G r_{n+1, k+1}\right)$, is equal to $\left[N_{1}\right]$. The last assertion follows from the fact that $x_{i}$ is linearly homotopic to the zero cross section. The same argument shows that $\theta$ is the Poincaré dual of any class of the form $\left[M_{v}\right]$, where $M_{v}$ is the submanifold of $A G r_{n, k}$ defined in the statement of Theorem 8 . We conclude that $\theta$, the Poincaré dual of $\left[N_{1}\right]=\left[X_{i}\right]=\left[M_{v}\right]$, is concentrated in an arbitrarily small open neighborhood $U_{i}$ of $X_{i}$, which means that $\theta$ is in the image of the map

$$
H^{n-k}\left(G r_{n+1, k+1}, G r_{n+1, k+1} \backslash U_{i}\right) \longrightarrow H^{n-k}\left(G r_{n+1, k+1}\right) .
$$

From here it follows, essentially by the argument of Theorem 11.10 in Chapter VI, p. 373, of [2], that $\theta^{l+1}$ is in the image of the map

$$
H^{(l+1)(n-k)}\left(G r_{n+1, k+1}, G r_{n+1, k+1} \backslash \bigcap_{i=0}^{l} U_{i}\right) \longrightarrow H^{(l+1)(n-k)}\left(G r_{n+1, k+1}\right),
$$

which means that $\theta^{l+1}$ is concentrated in an arbitrarily small neighborhood of the set $C_{k}(\mathcal{K})$, in the sense of Definition 1 .

In the proof of Corollary 1 we assume that the cohomology we work with is a continuous extension of the singular cohomology theory, say the Čech or the AlexanderSpanier-Kolmogoroff cohomology theory. This is only a technical assumption, similar to the corresponding assumptions in Poincaré-Lefschetz duality theorems. 
Proof of Corollary 1. Suppose that $\operatorname{Cat}_{G r}\left(C_{k}(\mathcal{K})\right) \leq k-l$. This means that there exists a family $\mathcal{F}=\left\{F_{1}, \ldots, F_{k-l}\right\}$ of closed subspaces of $G r=G r_{n+1, k+1}$ such that $\bigcup_{j=1}^{k-l} F_{j}=C_{k}(\mathcal{K})$ and each $F_{i}$ is contractible to a point by a homotopy which moves $F_{i}$ inside $G r$. More precisely, this means that the inclusion map $F_{i} \hookrightarrow G r$ is homotopic to a constant map and as a consequence of this, the induced map $H^{n-k}\left(G r, Z_{2}\right) \rightarrow$ $H^{n-k}\left(F_{i}, Z_{2}\right)$ is trivial. Moreover, by the continuity of the cohomology theory, there exists an open set $V_{i} \supset F_{i}$ such that the homomorphism $H^{n-k}\left(G r, Z_{2}\right) \rightarrow H^{n-k}\left(V_{i}, Z_{2}\right)$ is also trivial. From the cohomology exact sequence of a pair we conclude that there exists a class $\theta_{i} \in H^{n-k}\left(G r, V_{i} ; Z_{2}\right)$ which is mapped to the class $\theta$ by the homomorphism $H^{n-k}\left(G r, F_{i} ; Z_{2}\right) \rightarrow H^{n-k}\left(G r, Z_{2}\right)$. By Theorem 8, the class $\theta^{l+1}$ is concentrated in an arbitrarily small neighborhood of $C_{k}(\mathcal{K})$. It follows that there exists a class $\theta_{0} \in H^{(n-k)(l+1)}\left(G r, G r \backslash V ; Z_{2}\right)$, where $V:=\bigcup_{i=1}^{k-l} V_{i}$, which is mapped to the class $\theta^{l+1}$. We conclude that the class $\theta_{0} \theta_{1} \cdots \theta_{k-l} \in H^{(n-k)(k+1)}\left(G r, G r ; Z_{2}\right) \cong 0$ is mapped to the class $\theta^{k+1}$. This is a contradiction to the fact from Theorem 8 , that $\theta^{k+1} \neq 0$.

Proof of Theorem 9. The key topological property of the set $C_{k}(\mathcal{K})$, used in the proof of Theorem 7, was the relation (1). We will show that the set $C$ has the same property, i.e., that

$$
\begin{aligned}
& \text { for each projective }(k-l-1) \text {-dimensional plane } P_{k-l-1} \subseteq R P^{n}, \\
& \qquad C \cap S_{k}\left(P_{k-l-1}\right) \neq \emptyset .
\end{aligned}
$$

After we establish (1a), the rest of the proof will be just the repetition of the proof of Theorem 7. So we concentrate on the proof of (1a). Given a $(k-l-1)$-dimensional plane $P_{k-l-1}$, let $M$ be the manifold of all projective $k$-dimensional planes $P$ such that $P_{k-l-1} \subseteq P$ and let $[M]$ be its fundamental class. Alternatively, the manifold $M$ can be described as the space of all linear $(k+1)$-dimensional subspaces in $R^{n+1}$ which contain the $(k-l)$-dimensional linear space $L_{k-l}$ associated to $P_{k-l-1}$. The Poincare dual of the class $[M]$ is the class $\theta^{k-l}$, where $\theta$ is the class introduced in the formulation of Theorem 8 . One way to see this is, in light of the fact that $\theta$ is the Poincaré dual of classes $\left[M_{v}\right]$ (see Theorem 8), to observe that $[M]=\left[M_{v_{1}}\right] \cap \cdots \cap\left[M_{v_{k-l}}\right]$, where $v_{i}$ are points which span $L_{k-l}$. In other words, the class $\theta^{l+1}$ is, by assumption, concentrated in an arbitrarily small neighborhood of $C$ and $\theta^{k-l}$, being a Poincaré dual, is concentrated in an arbitrarily small neighborhood of the manifold $M$. Since the nonzero class $\theta^{k+1}=\theta^{l+1} \theta^{k-l}$ is concentrated in an arbitrarily small neighborhood of $C \cap M$, we conclude that this set is nonempty and the relation (1a) follows. Note that this proof is essentially a repetition of the argument already used in the proof of Corollary 1, see also the proof of Theorem 11.10 in Chapter VI, p. 373, of [2].

\section{Counterexamples for Nonconvex Bodies}

One can ask if one could replace the convex bodies $K_{i}$ with more general, say compact, sets. However, for one-dimensional sections this is not the case, as shown by an example kindly communicated to us by J. Matoušek. 
Example 1 (J. Matoušek). The geometrical form of Theorem 1, without assuming $0 \in$ int $K$ (and hence the stronger Theorem 3), is false for the compact set $K=\{x \in$ $\left.R^{n} \mid r_{1} \leq\|x\| \leq r_{2}\right\}$, where $0<r_{1}<r_{2}$ (and for $K_{1}=K$ and $K_{2}$ the unit ball of $R^{n}$, respectively). In particular, for $n=2$ the (geometrical forms) of Theorems 1-8 and Corollary 1 (without assuming $0 \in$ int $K$ at Theorems 1 and 2), with the maximal $k$-sections, are not valid for all compact sets.

Proof. If a line $A_{1}$ in $R^{n}$ has a distance $x$ from 0 , then

$$
V_{1}\left(K \cap A_{1}\right)=2\left(\sqrt{\max \left(r_{2}^{2}-x^{2}, 0\right)}-\sqrt{\max \left(r_{1}^{2}-x^{2}, 0\right)}\right),
$$

that attains its maximum for $x=r_{1}$. Hence all maximal 1-sections are those with $A_{1}$ being tangent to the ball of radius $r_{1}$ about 0 , and none of these lines $A_{1}$ contains 0 .

Based on this example, we can give further examples, for $k$-dimensional sections, $1 \leq$ $k \leq n-1$.

Example 2. For each $k \in\{1, \ldots, n-1\}$ the statement of Theorem 6 (and that of its special case Theorem 5) is not valid for all compact sets $K_{i}$.

Proof. Let $K_{1}=K \times B^{k-1}$, where $K=\left\{x \in \operatorname{lin}\left\{e_{1}, \ldots, e_{n-k+1}\right\} \mid r_{1} \leq\|x\| \leq r_{2}\right\}$ (with $0<r_{1}<r_{2}$ ) and $B^{k-1}=\left\{x \in \operatorname{lin}\left\{e_{n-k+2}, \ldots, e_{n}\right\} \mid\|x\| \leq 1\right\}$, and let $K_{2}, \ldots, K_{k+1}$ be $k$ unit balls centered at $0, e_{n-k+2}, \ldots, e_{n}$. Let $A_{k} \subseteq R^{n}$ be an affine $k$-dimensional plane, and suppose that $K_{i} \cap A_{k}$ is a maximal $k$-section of $K_{i}$ for any $i \in\{2, \ldots, k+1\}$. Then $A_{k}$ passes through the centers of $K_{2}, \ldots, K_{k+1}$, hence is of the form $A_{k}=A_{1} \times \operatorname{lin}\left\{e_{n-k+2}, \ldots, e_{n}\right\}$, where $0 \in A_{1} \subseteq \operatorname{lin}\left\{e_{1}, \ldots, e_{n-k+1}\right\}$ is a linear onedimensional subspace. Then, by Example $1, V_{k}\left(K_{1} \cap A_{k}\right)=V_{1}\left(K \cap A_{1}\right) \cdot V_{k-1}\left(B^{k-1}\right)<$ $V_{1}\left(K \cap\left(A_{1}+x\right)\right) \cdot V_{k-1}\left(B^{k-1}\right)=V_{k}\left(K_{1} \cap\left(A_{k}+x\right)\right)$ for $x \in \operatorname{lin}\left\{e_{1}, \ldots, e_{n-k+1}\right\}$ orthogonal to $A_{1}$ and $\|x\|=r_{1}$, hence $K_{1} \cap A_{k}$ is not a maximal $k$-section of $K_{1}$.

\section{Acknowledgments}

The authors would like to express their gratitude to Imre Bárány, organizer of a miniconference on topological methods in combinatorics and convexity (Budapest, November 1996), where this work began, to J. Matoušek for contributing Example 1, to R. Howard for communicating to us the proof of the integral geometric formula (3) for the $\left(\mathcal{H}^{v}, v\right)$ rectifiable and $\mathcal{H}^{\nu}$-measurable case and kindly agreeing to include it in this paper, and to the referees for their detailed remarks improving this paper. (One referee also noted the extendability of our results to the $\left(\mathcal{H}^{v}, v\right)$-rectifiable and $\mathcal{H}^{v}$-measurable case. Our original version of Theorems 7 and 9 concerned only the $C^{\infty}$ case.)

\section{References}

1. T. Bonnesen and W. Fenchel, Theorie der konvexen Körper, Springer-Verlag, Berlin, 1934.

2. G. E. Bredon, Topology and Geometry, Springer-Verlag, New York, 1993. 
3. J. E. Brothers, Integral geometry in homogenous spaces, Trans. Amer. Math. Soc. 124 (1966), 480-517.

4. V. L. Dol'nikov, Transversals of families of sets in $R^{n}$ and a connection between the Helly and Borsuk theorems, Russian Acad. Sci. Sb. Math. 79(1) (1994), 93-107.

5. E. Fadell and S. Husseini, An ideal-valued cohomological index theory with applications to Borsuk-Ulam and Bourgin-Yang theorems, Ergodic Theory Dynamical Systems 8 (1988), 73-85.

6. H. Federer, Geometric Measure Theory, Springer-Verlag, New York, 1969.

7. R. J. Gardner, Geometric Tomography, Cambridge University Press, Cambridge, 1996.

8. P. Griffiths and J. Harris, Principles of Algebraic Geometry, Wiley-Interscience, New York, 1978.

9. P. C. Hammer, Convex bodies associated with a convex body, Proc. Amer. Math. Soc. 2 (1951), 781-793.

10. P. C. Hammer, Convex curves of constant Minkowski breadth, in Convexity (Proc. Symposia in Pure Math. 7) (V. Klee, ed.), Amer. Math. Soc., Providence, RI, 1963, pp. 291-304.

11. R. Howard, The Kinematic Formula in Riemannian Homogeneous Spaces, Memoirs of the Amer. Math. Soc. 509, Amer. Math. Soc., Providence, RI, 1993.

12. E. Lutwak, Intersection bodies and dual mixed volumes, Adv. in Math. 71 (1988), 232-261.

13. E. Makai, Jr., and H. Martini, On bodies associated with a given convex body, Canad. Math. Bull. 39 (1996), 448-459.

14. E. Makai, Jr., and H. Martini, On maximal $k$-sections and related common transversals of convex bodies, Canad. Math. Bull., to appear.

15. H. Martini, Extremal equalities for cross-sectional measures of convex bodies, Proc. of the Third Geometry Congress (Thessaloniki, 1991), Aristoteles University Press, Thessaloniki, 1992, pp. 285-296.

16. J. Milnor and J. D. Stasheff, Characteristic Classes, Princeton University Press, Princeton, NJ, 1974.

17. C. M. Petty and J. M. Crotty, Characterizations of spherical neighborhoods, Canad. J. Math. 22 (1970), 431-435.

18. E. F. Whittlesey, Fixed points and antipodal points, Amer. Math. Monthly 70 (1963), 807-821.

19. R. Živaljević, Topological methods, in Handbook of Discrete and Computational Geometry (J. E. Goodman and J. O'Rourke, eds.), CRC Press, New York, 1997, pp. 209-224.

20. R. Živaljević and S. Vrećica, An extension of the ham-sandwich theorem, Bull. London Math. Soc. 22 (1990), 183-186.

Received May 22, 1998, and in revised form March 27, 2000. Online publication September 22, 2000. 